\title{
A prospective study of endometriosis and risk of type 2 diabetes
}

\author{
Leslie V. Farland ${ }^{1}$ (D) - William J. Degnan ${ }^{2}$ (D) $\cdot$ Holly R. Harris ${ }^{3,4}$ (D) $\cdot{\text { Deirdre K. } \text { Tobias }^{5,6} \text { (D) Stacey A. Missmer }}^{7,8}$ (D)
}

Received: 15 May 2020 / Accepted: 26 October 2020 / Published online: 5 January 2021

(C) The Author(s), under exclusive licence to Springer-Verlag GmbH, DE part of Springer Nature 2021

\begin{abstract}
Aims/hypothesis The objective of this study was to investigate the association between laparoscopically confirmed endometriosis and risk of type 2 diabetes.

Methods We used data from the Nurses' Health Study II, a prospective cohort of female nurses followed for $>25$ years $(N=$ 112,037). We used Cox proportional hazards models to estimate the HRs and 95\% CIs of incident, confirmed type 2 diabetes ( $n=8496$ participants) adjusted a priori for confounding factors. We additionally investigated differences in the relationship between endometriosis and type 2 diabetes by age ( $<50$ or $\geq 50$ years), BMI ( $<30$ or $\left.\geq 30 \mathrm{~kg} / \mathrm{m}^{2}\right)$, infertility history, menopausal status and history of gestational diabetes mellitus (GDM; restricted to parous women).

Results We saw no association between laparoscopically confirmed endometriosis and risk of type 2 diabetes in multivariable confounder-adjusted models (HR 1.06 [95\% CI 0.98, 1.13]) or models accounting for potential mediating factors (HR 0.94 [95\% CI $0.87,1.00]$ ). However, we observed modest differences in the association between endometriosis and type 2 diabetes by BMI group, history of infertility and history of GDM. Among non-obese women (HR 1.17 [95\% CI 1.02, 1.35]), women who never experienced infertility (HR 1.14 [95\% CI 1.04, 1.25]) and women who never experienced GDM (HR 1.11 [95\% CI 1.01, 1.22]), endometriosis was associated with greater risk of type 2 diabetes.

Conclusions/interpretation Overall, women with endometriosis were not at increased risk of type 2 diabetes. However, among subgroups at low risk for type 2 diabetes (i.e. non-obese women and women with no prior history of infertility or GDM), endometriosis was associated with a modest increased risk of type 2 diabetes.
\end{abstract}

Keywords Diabetes $\cdot$ Endometriosis $\cdot$ Epidemiology $\cdot$ Gestational diabetes

\section{Abbreviations}

GDM Gestational diabetes mellitus

Leslie V. Farland

1farland@email.arizona.edu

1 Department of Epidemiology and Biostatistics, Mel and Enid Zuckerman College of Public Health, University of Arizona, Tucson, AZ, USA

2 Department of Community, Environment, and Policy, Mel and Enid Zuckerman College of Public Health, University of Arizona, Tucson, AZ, USA

3 Epidemiology, Public Health Science Division, Fred Hutchinson Cancer Research Center, Seattle, WA, USA
NHSII Nurses' Health Study II

OC Oral contraceptive

4 Department of Epidemiology, University of Washington, Seattle, WA, USA

5 Department of Nutrition, Harvard T.H. Chan School of Public Health, Boston, MA, USA

6 Division of Preventive Medicine, Brigham and Women's Hospital and Harvard Medical School, Boston, MA, USA

7 Department of Epidemiology, Harvard T.H. Chan School of Public Health, Boston, MA, USA

8 Department of Obstetrics, Gynecology, and Reproductive Biology, College of Human Medicine, Michigan State University, Grand Rapids, MI, USA 


\section{Research in context}

\section{What is already known about this subject?}

- Prior research has suggested that women with endometriosis are at greater risk of cardiometabolic conditions (i.e. hypertension and hypercholesterolaemia)

- The relationship between endometriosis and risk of type 2 diabetes is unknown

\section{What is the key question?}

- Is endometriosis associated with risk of type 2 diabetes?

\section{What are the new findings?}

- Among women who were considered traditionally low risk for type 2 diabetes (e.g. not obese or who never experienced gestational diabetes), endometriosis was associated with a modest increased risk of type 2 diabetes

How might this impact on clinical practice in the foreseeable future?

- To our knowledge, this is the first study to prospectively investigate the relationship between endometriosis and risk of type 2 diabetes. It suggests that endometriosis may increase type 2 diabetes risk among women traditionally considered to be at low risk

\section{Introduction}

Endometriosis is a chronic, inflammatory, oestrogen-dependent disease characterised by the presence of endometrial-like tissue outside the uterus, mainly within the peritoneal cavity [1]. It affects $\sim 10 \%$ of women in their reproductive years, translating to 176 million women worldwide, and is associated with pelvic pain and infertility [2]. Research suggests an association between endometriosis and the subsequent development of other chronic cardiometabolic conditions [3, 4]. Prior research has suggested that women with endometriosis may be at greater risk of hypertension [5], hypercholesterolaemia [5-8] and subclinical atherosclerosis [9]. Additionally, prior research from the Nurses' Health Study II (NHSII) observed that women with endometriosis had a $60 \%$ higher risk of CVD (myocardial infarction, angiographically confirmed angina or coronary artery bypass graft), with the highest risk among young women $(\leq 50$ years old $)[10]$.

Endometriosis may influence type 2 diabetes risk through a variety of mechanisms. Women with endometriosis have a hyperinflammatory milieu both locally (in the peritoneal cavity) and systemically $[11,12]$. Research has found that several inflammatory markers are elevated [13] in women with endometriosis, including intracellular adhesion molecule 1, Creactive protein, IL-1, IL-6, TNF- $\alpha$ and vascular endothelial growth factor $[14,15]$. Additionally, endometriosis status is correlated with decreased levels of adiponectin [16] and increased levels of leptin [17] compared with women without the disease, although these findings have been inconsistent [18].

Understanding the relationship between endometriosis and type 2 diabetes is critical to public health, given the increasing prevalence of type 2 diabetes. Currently, 9.4\% of the U.S. population is diagnosed with type 2 diabetes [19]. Prior research suggests that there may be an association between laparoscopically confirmed endometriosis diagnosis and risk of gestational diabetes, and that the risk may vary by history of infertility $[20,21]$. Thus, we propose to investigate the relationship between endometriosis and risk of type 2 diabetes in the NHSII, a prospective cohort with detailed information on laparoscopically confirmed endometriosis history, incident diabetes diagnoses and potential confounding and modifying factors. Additionally, given previously observed effect modification for relationships with endometriosis by age/ menopausal status [1, 10], infertility history [21, 22] and body size $[23,24]$, and an established relationship between obesity and history of gestational diabetes with type 2 diabetes, we will investigate whether the association between endometriosis and risk of type 2 diabetes differs when stratified by age, menopausal status, infertility history, BMI and history of gestational diabetes.

\section{Methods}

Study population The NHSII is an ongoing prospective cohort of 116,429 female registered nurses who were 25-42 years of age at enrolment in 1989. At baseline in 1989 and every 2 years thereafter, participants completed self-administered questionnaires to capture detailed information on a variety of lifestyle and reproductive characteristics, and to update healthrelated outcomes. Cumulative follow-up of the cohort is $>90 \%$. Of the 116,429 women initially enrolled in the Nurses' Health Study, women who reported a diagnosis of diabetes (type 1 or type 2), stroke or myocardial infarction, 
or who received a coronary artery bypass graft, prior to June 1989 when the cohort began were excluded from our analysis. This left 112,037 women followed from 1989 until return of the final follow-up questionnaire (by June 2017) available for the present study. The NHSII protocol was approved by the Institutional Review Board of the Partners Health Care System, Boston, MA, USA and participants gave informed consent.

Endometriosis diagnosis Women were asked on each biennial questionnaire from 1993 onwards whether they had physiciandiagnosed endometriosis. Participants who responded 'yes' indicated the year of diagnosis and whether it had been confirmed by laparoscopy, the clinical gold standard for endometriosis diagnosis [25-27]. Self-reported endometriosis was validated among a random subgroup of NHSII participants $(n=200)$. For women whose medical records were available, a diagnosis of endometriosis was confirmed in $96 \%$ of women reporting laparoscopically confirmed endometriosis, but in only $54 \%$ of women without laparoscopic confirmation [28]. Because of the potential for misclassification by women with self-reported endometriosis without laparoscopic confirmation, we restricted our endometriosis definition to laparoscopically confirmed endometriosis. Endometriosis diagnosis status was treated as time-varying and updated over time. Once a woman reported laparoscopically confirmed endometriosis, she was considered to have exposure to a history of endometriosis through the remainder of follow-up. At baseline in 1989, 5242 women had a history of endometriosis diagnosis and 7061 women were diagnosed with endometriosis after cohort enrolment in 1989.

Type 2 diabetes Type 2 diabetes diagnoses were ascertained on the biennial questionnaire based on self-reported physician diagnosis. Women who responded positively were sent a supplemental questionnaire and cases were confirmed if they met the National Diabetes Data Group classification [29]: at least one classic symptom (excessive thirst, polyuria, unintentional weight loss or hunger) and a fasting plasma glucose concentration of $\geq 7.8 \mathrm{mmol} / \mathrm{l}(140 \mathrm{mg} / \mathrm{dl})$ or random plasma glucose concentration of $\geq 11.1 \mathrm{mmol} / 1$ ( $200 \mathrm{mg} / \mathrm{dl})$; no symptoms but at least a twofold elevation in plasma glucose concentration on more than one occasion (fasting plasma glucose $\geq 7.8 \mathrm{mmol} / 1$, random plasma glucose $\geq 11.1 \mathrm{mmol} / 1$ or $2 \mathrm{~h} \mathrm{OGTT} \mathrm{of} \geq 11.1 \mathrm{mmol} / \mathrm{l}$ ); or glucose-lowering medication use (insulin or oral glucose-lowering agent). In 1998, the diagnostic criteria changed to adopt a new diagnostic threshold for fasting plasma glucose of $\geq 7.0 \mathrm{mmol} / \mathrm{l}(126 \mathrm{mg} / \mathrm{dl})$ [30]. The similar Nurses' Health Study cohort found high accuracy comparing this validation technique against medical records (98\%) [31]. At the end of follow-up, there were 8496 incident cases of type 2 diabetes across 2,691,401 personyears of follow-up.
Table 1 Age-standardised characteristics of the NHSII study population at baseline, in 1989, by laparoscopically confirmed endometriosis diagnosis

\begin{tabular}{|c|c|c|}
\hline \multirow[t]{2}{*}{ Characteristic } & \multicolumn{2}{|c|}{ Confirmed endometriosis } \\
\hline & Yes $(n=5242)$ & No $(n=106,795)$ \\
\hline Age, years ${ }^{\mathrm{a}}$ & $36.0(4.2)$ & $34.7(4.7)$ \\
\hline \multicolumn{3}{|c|}{ BMI at 18 years old $\left(\mathrm{kg} / \mathrm{m}^{2}\right), \%$} \\
\hline$<18.5$ & 19 & 15 \\
\hline $18.5-<22.5$ & 61 & 61 \\
\hline $22.5-<25$ & 13 & 14 \\
\hline$\geq 25$ & 8 & 11 \\
\hline \multicolumn{3}{|l|}{$\mathrm{BMI}$ at baseline $\mathrm{kg} / \mathrm{m}^{2}, \%$} \\
\hline$<18.5$ & 4 & 3 \\
\hline $18.5-22.5$ & 45 & 44 \\
\hline $22.5-<25$ & 23 & 22 \\
\hline $25-<30$ & 19 & 19 \\
\hline$\geq 30$ & 9 & 12 \\
\hline \multicolumn{3}{|c|}{ Age at menarche, years, $\%$} \\
\hline$\leq 11$ years old & 29 & 24 \\
\hline $12-13$ years old & 56 & 58 \\
\hline 14+ years old & 15 & 18 \\
\hline \multicolumn{3}{|l|}{ History of infertility } \\
\hline Yes, \% & 54 & 17 \\
\hline \multicolumn{3}{|l|}{ OC status, $\%$} \\
\hline Never & 11 & 17 \\
\hline Past & 80 & 70 \\
\hline Current & 10 & 13 \\
\hline \multicolumn{3}{|l|}{ Smoking status, $\%$} \\
\hline Never & 65 & 66 \\
\hline Past & 21 & 21 \\
\hline Current & 14 & 13 \\
\hline \multicolumn{3}{|c|}{ Cumulative average physical activity (met-h/week), \% } \\
\hline $0-3$ & 18 & 18 \\
\hline $3-9$ & 27 & 27 \\
\hline $9-<18$ & 26 & 25 \\
\hline $18-<27$ & 16 & 16 \\
\hline $27+$ & 13 & 14 \\
\hline \multicolumn{3}{|l|}{ Race/ethnicity, \% } \\
\hline White, non-Hispanic & 94 & 93 \\
\hline Other race/ethnicity & 6 & 7 \\
\hline \multicolumn{3}{|c|}{ History of hypercholesterolaemia } \\
\hline Yes, $\%$ & 13 & 10 \\
\hline \multicolumn{3}{|l|}{ History of hypertension } \\
\hline Yes, $\%$ & 6 & 5 \\
\hline \multicolumn{3}{|c|}{ Family history of diabetes ${ }^{\mathrm{b}}$} \\
\hline Yes, $\%$ & 18 & 17 \\
\hline \multicolumn{3}{|c|}{ History of gestational diabetes } \\
\hline Yes, \% & 3 & 3 \\
\hline
\end{tabular}

Values are means (SD) for continuous variables; percentages for categorical variables; and are standardised to the age distribution of the study population

Values of polytomous variables may not sum to $100 \%$ because of rounding

${ }^{\text {a }}$ Value is not age-adjusted

${ }^{\mathrm{b}}$ NHSII questionnaires do not specify type of diabetes in family members

Covariate data On the 1989 baseline questionnaire, participants reported a number of characteristics including their height, current weight, weight at age 18, physical activity, smoking history, age at menarche, oral contraceptive (OC) use, parity (number of pregnancies lasting $\geq 6$ months), history of infertility ( $>12$ months trying to conceive without success), family history 
of diabetes, physician-diagnosed type 2 diabetes, physiciandiagnosed CVD, physician-diagnosed cancer, physiciandiagnosed elevated cholesterol, physician-diagnosed elevated BP, physician-diagnosed gestational diabetes, menopausal status, history of bilateral oophorectomy, and their race and ethnicity. Information on race and ethnicity was asked for in 1989 and 2005; if missing ( 1.6\%), information was imputed based on geocoded location for reporting to the National Institutes of Health. All time-varying characteristics were updated every 2-4 years. For each covariate in our multivariable models we outline below our approach to handling missing data, the frequency of missingness and whether the missing indicator was associated with the outcome. Overall missingness for covariates was minimal ( $<1 \%$ for most variables). Broadly, for covariates with missing data, data were carried forward from previous questionnaires if available and a missing indicator variable was created, where appropriate.

Statistical analyses Person-months at risk were calculated from entry into the cohort in 1989 until confirmation of: (1) death; (2) type 2 diabetes diagnosis; or (3) their last returned questionnaire, whichever came first. To account for possible confounding by age, all variables presented in Table 1 (aside from age) were standardised to the age distribution of the study population. Cox proportional hazards models stratified by calendar time (years) with age (months) as the time metameter were used to calculate the HRs and 95\% CIs of incident type 2 diabetes diagnosis (model 1). The proportional hazards assumptions were tested using the likelihood-ratio test comparing a model with and without an interaction term for time, and they were met. Known a priori potential confounders for endometriosis and type 2 diabetes were adjusted for [32], with time-varying covariates updated biennially at every questionnaire cycle (model 2 ): BMI at age 18 $\left(\mathrm{kg} / \mathrm{m}^{2}\right)(<18.5,18.5-<22.5,22.5-<25,25-<30,30-<35,35-$ $<40$ or $40+$; missing indicator created; cumulative per cent missing: $0.99 \%, p=0.84$ for missing indicator associated with type 2 diabetes), current BMI $(<18.5,18.5-<22.5,22.5-<25$, $25-<30,30-<35,35-<40,40+$; missing indicator created; $0.17 \%, p<0.01)$, age at menarche $(\leq 11,12-13, \geq 14$ years old; missing indicator created; $0.33 \%, p=0.93$ ), history of infertility (yes or no; missing indicator created; no individual missing information across every questionnaire; $p=0.91$ ), OC status history (never, past or current; missing indicator created; $<0.1 \%, p=0.81$ ), smoking history (never, past or current; missing indicator created; $<0.1 \%, p=0.60)$, physical activity $(0-<3$ metabolic equivalent-hours/week, 3-<9, 9-<18, 18$<27, \geq 27$; missing indicator created; $<0.1 \%, p=0.92$ ) and race/ethnicity (white or non-white). Multivariable model 3 additionally adjusted for known clinical risk factors for type 2 diabetes that maybe considered mediators [33] and may be on the causal pathway between endometriosis and risk of type 2 diabetes: history of hypercholesterolaemia (yes or no; based on questionnaire structure missingness cannot be quantified), history of hypertension (yes or no; based on questionnaire structure missingness cannot be quantified), family history of diabetes (yes or no; based on questionnaire structure missingness cannot be quantified), history of gestational diabetes (yes or no; based on questionnaire structure missingness cannot be quantified), menopausal status (premenopausal, postmenopausal or perimenopausal/unsure; missing indicator created; no individual missing information across every questionnaire, $p=0.50$ ) and history of bilateral oophorectomy (yes or no; missing indicator created; no individual missing information across every questionnaire, $p=0.09$ ).

Given effect modification by age and infertility history observed in prior analyses of endometriosis, and established risk for type 2 diabetes by obesity and gestational diabetes, we assessed heterogeneity by potential effect modifiers, including current age $(<50$ or $\geq 50$ years old) (based on mean age at menopause for women [34]), menopausal status (pre- or postmenopausal), BMI ( $<30$ or $\geq 30 \mathrm{~kg} / \mathrm{m}^{2}$ ), history of infertility (yes or no) and history of gestational diabetes (yes or no; analyses restricted to parous women). These categorisations were time-varying and women contributed person-time to the appropriate strata given their status over follow-up. Likelihood-ratio tests were used to test for statistically significant differences among groups on the multiplicative scale. This was done by comparing multivariable adjusted models (model 2) with and without an interaction term.
Table 2 Laparoscopically confirmed endometriosis diagnosis in relation to risk of type 2 diabetes in the NHSII

\begin{tabular}{lllll}
\hline Endometriosis & $\begin{array}{l}\text { Cases/person- } \\
\text { years }\end{array}$ & HR of incident type 2 diabetes (95\% CI) & \\
\cline { 3 - 5 } & Model 1 & Model 2 & Model 3 \\
\hline No & $7543 / 2,448,284$ & 1.0 (referent) & 1.0 (referent) & 1.0 (referent) \\
Yes & $953 / 243,117$ & $1.11(1.04,1.19)$ & $1.06(0.98,1.13)$ & $0.94(0.87,1.00)$ \\
\hline
\end{tabular}

Model 1: adjusted for age (months) and calendar time

Model 2: additionally adjusted for BMI at age 18, BMI current, age at menarche, history of infertility, OC use history, smoking history, physical activity and race

Model 3: additionally adjusted for history of elevated cholesterol, history of hypertension, family history of diabetes, history of gestational diabetes, menopausal status and history of bilateral oophorectomy 
Several additional a priori sensitivity analyses were conducted to investigate the magnitude of potential biases: (1) to investigate the possible role of selection bias, we excluded prevalent endometriosis diagnosed before cohort enrolment (1989); (2) to address the potential diagnostic delay between endometriosis symptom onset and surgical diagnosis [35, 36], the reported diagnostic date of endometriosis was pre-dated by 4,6 and 8 years in sensitivity analyses to investigate the effect of this
Table 3 Laparoscopically confirmed endometriosis in relation to type 2 diabetes risk in the NHSII stratified by age, menopausal status, BMI, history of infertility and history of gestational diabetes

\begin{tabular}{|c|c|c|c|c|}
\hline \multirow[t]{2}{*}{ Endometriosis } & \multirow[t]{2}{*}{ Cases/person-years } & \multicolumn{2}{|c|}{ HR for incident type 2 diabetes $(95 \% \mathrm{CI})$} & \multirow[t]{2}{*}{$\mathrm{p}$ value } \\
\hline & & Model $1^{\mathrm{a}}$ & Model $2^{\mathrm{b}}$ & \\
\hline \multicolumn{5}{|c|}{ Stratified by age } \\
\hline \multicolumn{5}{|c|}{$<50$ years old } \\
\hline No & $3261 / 1,575,674$ & 1.0 (referent) & 1.0 (referent) & \\
\hline Yes & $352 / 133,918$ & $1.08(0.97,1.21)$ & $1.09(0.97,1.22)$ & 0.59 \\
\hline \multicolumn{5}{|c|}{$\geq 50$ years old } \\
\hline No & $4282 / 872,611$ & 1.0 (referent) & 1.0 (referent) & \\
\hline Yes & $601 / 109,199$ & $1.13(1.04,1.23)$ & $1.06(0.97,1.15)$ & \\
\hline \multicolumn{5}{|c|}{ Stratified by menopausal status } \\
\hline \multicolumn{5}{|c|}{ Premenopausal } \\
\hline No & $3330 / 1,607,710$ & 1.0 (referent) & 1.0 (referent) & \\
\hline Yes & $204 / 100,320$ & $0.93(0.81,1.07)$ & $1.07(0.92,1.23)$ & 0.42 \\
\hline \multicolumn{5}{|c|}{ Postmenopausal } \\
\hline No & $3602 / 730,315$ & 1.0 (referent) & 1.0 (referent) & \\
\hline Yes & $677 / 128,436$ & $1.11(1.02,1.21)$ & $1.08(0.99,1.18)$ & \\
\hline \multicolumn{5}{|c|}{ Stratified by BMI } \\
\hline \multicolumn{5}{|c|}{$<30 \mathrm{~kg} / \mathrm{m}^{2}$} \\
\hline No & $1635 / 1,702,054$ & 1.0 (referent) & 1.0 (referent) & \\
\hline Yes & $245 / 168,105$ & $1.31(1.14,1.50)$ & $1.17(1.02,1.35)$ & 0.01 \\
\hline \multicolumn{5}{|l|}{$\geq 30 \mathrm{~kg} / \mathrm{m}^{2}$} \\
\hline No & $4940 / 452,048$ & 1.0 (referent) & 1.0 (referent) & \\
\hline Yes & $579 / 48,084$ & $1.02(0.93,1.11)$ & $1.00(0.91,1.09)$ & \\
\hline \multicolumn{5}{|c|}{ Stratified by history of infertility } \\
\hline \multicolumn{5}{|c|}{ No infertility } \\
\hline No & $5531 / 1,939,493$ & 1.0 (referent) & 1.0 (referent) & \\
\hline Yes & $503 / 115,809$ & $1.26(1.15,1.38)$ & $1.14(1.04,1.25)$ & 0.02 \\
\hline \multicolumn{5}{|l|}{ Infertile } \\
\hline No & $2012 / 508,791$ & 1.0 (referent) & 1.0 (referent) & \\
\hline Yes & $450 / 127,308$ & $0.86(0.77,0.95)$ & $0.98(0.89,1.09)$ & \\
\hline \multicolumn{5}{|c|}{ Stratified by history of gestational diabetes ${ }^{\mathrm{d}}$} \\
\hline \multicolumn{5}{|c|}{ No history of gestational diabetes } \\
\hline No & $4587 / 1,659,468$ & 1.0 (referent) & 1.0 (referent) & \\
\hline Yes & $527 / 134,447$ & $1.23(1.13,1.35)$ & $1.11(1.01,1.22)$ & 0.02 \\
\hline \multicolumn{5}{|c|}{ History of gestational diabetes } \\
\hline No & $829 / 80,765$ & 1.0 (referent) & 1.0 (referent) & \\
\hline Yes & $86 / 8131$ & $0.96(0.75,1.20)$ & $0.93(0.73,1.19)$ & \\
\hline
\end{tabular}

${ }^{\mathrm{a}}$ Model 1: adjusted for age (months) and calendar time

${ }^{\mathrm{b}}$ Model 2: additionally adjusted for BMI at age 18, BMI current, age at menarche, history of infertility, OC use history, smoking history, physical activity, race and bilateral oophorectomy (analyses stratified by BMI and infertility)

${ }^{\mathrm{c}} p$ value, likelihood-ratio test for heterogeneity between groups

${ }^{\mathrm{d}}$ Analyses restricted to parous women 
temporal misclassification of exposure; (3) in sensitivity analyses for our exposure definition, we expanded our endometriosis definition to include disease diagnoses with and without laparoscopic confirmation; and (4) in sensitivity analyses for our type 2 diabetes, we restricted to type 2 diabetes diagnosed during screening, not based on symptoms.

\section{Results}

At cohort baseline in 1989, women with laparoscopically confirmed endometriosis were less likely to be overweight at age 18 (BMI $\geq 25: 8 \%$ vs $11 \%$ ), were less likely to be obese in 1989 (BMI $\geq 30: 9 \%$ vs $12 \%$ ) and were more likely to experience a younger age at menarche ( $\leq 11: 29 \%$ vs $24 \%)$ compared with women without endometriosis (Table 1). Women with endometriosis were more likely to report a history of infertility (54\% vs $17 \%$ ) and to be past or current OC users (90\% vs $83 \%$ ) compared with women without endometriosis. There were no meaningful differences between women with and without endometriosis in history of hypertension (6\% vs $5 \%$ ), history of hypercholesterolaemia (13\% vs $10 \%)$ or family history of type 2 diabetes ( $18 \%$ vs $17 \%$ ) at baseline.

In age and calendar time-adjusted models, women with a history of laparoscopically confirmed endometriosis had a modest risk of type 2 diabetes (HR 1.11 [95\% CI 1.04, 1.19]) compared with women with no history of endometriosis (Table 2). However, this was attenuated after adjustment for potential confounding factors (HR 1.06 [95\% CI $0.98,1.13]$ ), which was driven by history of infertility and age at menarche. The association further attenuated in analyses adjusted for clinical type 2 diabetes risk factors or covariates that may be on the causal pathway between endometriosis and type 2 diabetes (HR 0.94 [95\% CI 0.87 , $1.00]$ ). Results did not meaningfully change in sensitivity analyses pre-dating endometriosis diagnoses, restricting endometriosis diagnoses to incident reports after cohort baseline in 1989, expanding endometriosis diagnoses to include non-laparoscopically confirmed disease and restricting type 2 diabetes diagnoses to those diagnosed via screening (data not shown).

We saw no differences in the associations between endometriosis and type 2 diabetes by age group $(<50$ years old vs $\geq 50$ years old; $p$ value, test for heterogeneity $=0.59$ ) or menopausal status ( $p$ value, test for heterogeneity $=0.42$ ) (Table 3). However, we did find differences by BMI ( $p$ value, test for heterogeneity $=0.01$ ), history of infertility ( $p$ value, test for heterogeneity $=0.02$ ) and history of gestational diabetes among parous women ( $p$ value, test for heterogeneity $=0.02$ ). Specifically, among non-obese women, endometriosis was associated with risk of type 2 diabetes (HR 1.17 [95\% CI 1.02, 1.35]), but there was no association among obese women (HR 1.00 [95\% CI 0.91,
1.09]). Among women who had never reported a history of infertility, there was a modest association between endometriosis and type 2 diabetes (HR 1.14 [95\% CI 1.04, 1.25]), but no association between endometriosis and type 2 diabetes among women reporting infertility (HR 0.98 [95\% CI $0.89,1.09])$. Lastly, we found that among parous women with no history of gestational diabetes, there was a modest association between endometriosis and risk of type 2 diabetes (HR: 1.11 [95\% CI 1.01, 1.22]). However, no association was found among parous women with prior history of gestational diabetes mellitus (GDM) (HR 0.93 [95\% CI $0.73,1.19])$.

\section{Discussion}

Overall, we did not observe an association between history of laparoscopically confirmed endometriosis and risk of type 2 diabetes after adjustment for common risk factors. However, the relationship between endometriosis and type 2 diabetes varied among subgroups. Specifically we found that women with endometriosis had a modest risk of type 2 diabetes among nonobese women, women with no history of infertility and women with no history of gestational diabetes, suggesting that the relationship between endometriosis and diabetes was strongest in subgroups with traditionally low type 2 diabetes risk.

Prior research on the relationships between reproductive factors and risk of type 2 diabetes has been limited. Previous research found that women with infertility had a $20 \%$ greater risk of developing type 2 diabetes compared with fertile women (HR 1.20 [95\% CI 1.14, 1.28]) [22]; however, there was no association between endometriosis as an indication for infertility and risk of diabetes (HR 1.06 [95\% CI 0.89, 1.27]). This is consistent with our findings that among women with a history of infertility, there was no association between endometriosis and risk of type 2 diabetes; however, we found that among women with no history of infertility, endometriosis was associated with a modest risk of type 2 diabetes (HR 1.14).

Women with endometriosis and no history of infertility may represent a unique endometriosis phenotype. Women surgically diagnosed with endometriosis who have never reported infertility are more likely to have presented clinically with pelvic pain symptoms, whereas women with endometriosis and infertility may have been diagnosed with endometriosis incidentally through an infertility evaluation [37]. We found that an association between endometriosis and type 2 diabetes existed among women who never experienced infertility. This mechanism could be driven by two pathways: (1) as prior research from this cohort indicates, women with infertility may already be at increased risk of type 2 diabetes [22], and thus the noninfertile group may be low risk for type 2 diabetes; and/or (2) infertility may be an informative phenotype for 
studying endometriosis. Ultimately, this informative heterogeneity requires additional research.

Women with endometriosis may undergo a variety of hormonal or surgical treatments (e.g. lesion excision, hysterectomy or oophorectomy) to alleviate symptoms that may contribute to type 2 diabetes risk [38]. A recent meta-analysis suggested that earlier age at menopause was associated with a higher risk of diabetes [39], for both natural and surgically induced menopause. Prior research on the relationship between surgically induced menopause through bilateral oophorectomy and risk of diabetes has been mixed [40]; however, some research has suggested that bilateral oophorectomy increases risk of both type 2 diabetes [41] and the metabolic syndrome [42]. To investigate the influence of surgical treatments that induce menopause, we adjusted for bilateral oophorectomy and menopausal status in multivariable model 3 , as these covariates may be on the causal pathway between endometriosis and type 2 diabetes [33]. Additionally, we stratified by age categories as well as menopausal status. Compared with model 2, which adjusted for potential confounding factors, multivariable model 3 was attenuated after adjustment for potential mediating variables, including age at menopause and bilateral oophorectomy, suggesting that these covariates may be contributing to the causal pathway between endometriosis and diabetes. We found no differences in the relationship between endometriosis and risk of type 2 diabetes according to age or menopausal status (Table 3); however, in our stratified analyses, we did observe that among lean women and fertile women, endometriosis was associated with diabetes. It is possible that the association between endometriosis and diabetes among women who are fertile may, in part, be mediated by endometriosis treatments that parous women are more likely to accept, such as oophorectomy. However, this hypothesis was not borne out by the data because adjustment for bilateral oophorectomy did not meaningfully change these results. Future research into the relationships between treatments for endometriosis and diabetes risk may further elucidate these mechanisms.

Among parous women, we also saw differences in the association between endometriosis and risk of type 2 diabetes by history of gestational diabetes. The observed association between endometriosis and type 2 diabetes in our cohort was limited to women with no prior history of gestational diabetes (HR 1.11). Gestational diabetes is an established risk factor for type 2 diabetes [43, 44], with women with gestational diabetes having a sevenfold greater risk of type 2 diabetes development compared with women who experienced a normoglycaemic pregnancy [45]. Thus, the potential influence of endometriosis on diabetes risk may be more impactful among low-risk women compared with higher-risk women. Prior research has found that endometriosis was associated with a higher risk of gestational diabetes [21], but only among women without a history of infertility [20], which is consistent with these results.
There is a strong and consistent inverse relationship between obesity and risk of endometriosis [1, 23, 24]. Indeed, in our study population, those with endometriosis were less likely to be obese at cohort baseline in 1989 and at 18 years old. Obesity is associated with insulin resistance [46] and is the strongest risk factor for development of type 2 diabetes [47]. In our study, we found that among non-obese, lean women, endometriosis was associated with a modest risk of diabetes (HR 1.17), but this association was not present among obese women (HR 1.00). This finding again suggests that among women who are at a lower-risk for type 2 diabetes, endometriosis emerges as a modest risk factor.

To our knowledge, we are the first study to prospectively investigate the relationship between endometriosis and risk of type 2 diabetes. We found that the relationship between endometriosis and type 2 diabetes was strongest among subgroups that have traditionally been considered low risk for type 2 diabetes. Additional research is necessary to confirm these findings.

This study has many strengths including its longitudinal follow-up, prospective ascertainment of endometriosis and incident type 2 diabetes, and large sample size with validated measures of type 2 diabetes and endometriosis diagnosis among a cohort of medical professionals. However, we also must recognise its limitations. There is a known diagnostic delay for many women experiencing endometriosis between symptom onset and disease diagnosis. On average, women with endometriosis may wait 7 years to receive a diagnosis and in our cohort women wait, on average, 4 years. This was investigated in sensitivity analyses where we pre-dated endometriosis diagnosis by 4,6 and 8 years and the results did not meaningfully change. Additionally, some members of the cohort may have not yet been diagnosed with endometriosis or may have asymptomatic disease. However, given the large number of women in our population without the disease, the inclusion of undiagnosed endometriosis in the truly unexposed group of women would likely have limited effect [48] and any potential bias would most likely attenuate our findings. Women with endometriosis may be more likely to be diagnosed with type 2 diabetes because they are better connected with the medical system through their endometriosis diagnosis and thus may see their healthcare provider more regularly allowing for more opportunities for a type 2 diabetes diagnosis. This potential bias is minimised by the fact that all participants were trained nurses and should be well connected with the medical system. Additionally, sensitivity analyses restricting to type 2 diabetes diagnoses via screening did not meaningfully change the results. The level of health education in this cohort of nursing professionals allows for high-quality information to be collected by self-report (as has been consistently demonstrated in validation studies) and reduces confounding by education and socioeconomic status. Treatments for endometriosis may mediate the association between endometriosis and risk of type 2 diabetes [38]. 
Unfortunately, within this large longitudinal cohort, we did not collect detailed information on hormonal treatments specifically for endometriosis. Multivariable models were adjusted for bilateral oophorectomy as a possible treatment for endometriosis, but we do not have information on whether the indication for oophorectomy was as a treatment for endometriosis. For covariates missing data in multivariable adjusted models, where appropriate, missingness was enumerated and data were carried forward from a prior questionnaire if available or a missing indicator variable was generated. For all covariates, except BMI, the missing indicator was not associated with type 2 diabetes. Given this relationship, creating a missing indicator variable for BMI may bias the results; however, we expect the bias to be minimal given the small number of individuals missing information on BMI $(0.17 \%)$.

Our findings suggest that overall, women with endometriosis are not at an increased risk of type 2 diabetes. However, among subgroups at low risk for type 2 diabetes (i.e. nonobese women and women with no prior history of infertility or gestational diabetes), endometriosis is associated with a modest increased risk of type 2 diabetes. As this is the first study to prospectively investigate the relationship between endometriosis and risk of type 2 diabetes, all findings should be replicated in additional study populations before inferring clinical and public health implications. Additionally, future research should emphasise exploration of potential effect modification by informative subgroups (e.g. age, BMI, infertility history and GDM) as well as treatments for endometriosis. Given that our study suggested that among women who have traditionally been believed to be at low risk for diabetes (e.g. lean or no history of GDM) endometriosis may confer increased risk, future research should explore the utility of targeted screening or intervention among these groups of women for whom endometriosis may be their only recognised risk factor for type 2 diabetes.

Acknowledgements The authors would like to acknowledge Channing Division of Network Medicine for their work maintaining the Nurses' Health Study data.

Data availability Further information including the procedures to obtain and access data from the Nurses' Health Studies and the Health Professionals Follow-up Study is described at https://www. nurseshealthstudy.org/researchers (contact email: nhsaccess@channing. harvard.edu) and https://sites.sph.harvard.edu/hpfs/for-collaborators/.

Funding This work was supported by an Endometriosis Foundation of America Research grant, grant U01 CA176726 and grant HD57210 from the Eunice Kennedy Shriver National Institute of Child Health and Human Development. HRH is supported by the National Cancer Institute, National Institutes of Health (K22 CA193860).

Authors' relationships and activities The authors declare that there are no relationships or activities that might bias, or be perceived to bias, their work.
Contribution statement LVF, WJD and SAM contributed to the conception and design, acquisition of data, and the analysis and interpretation of data; drafting the article and revising it critically for important intellectual content; and approved the final version for publication. HRH and DKT contributed to the study design and interpretation of data; revised the article critically for important intellectual content; and approved the final version for publication. LVF is responsible for the integrity of the work as a whole.

\section{References}

1. Shafrir AL, Farland LV, Shah DK et al (2018) Risk for and consequences of endometriosis: a critical epidemiologic review. Best Pract Res Clin Obstet Gynaecol. https://doi.org/10.1016/j. bpobgyn.2018.06.001

2. Zondervan KT, Becker CM, Koga K, Missmer SA, Taylor RN, Vigano P (2018) Endometriosis. Nat Rev Dis Primers 4(1):9. https://doi.org/10.1038/s41572-018-0008-5

3. Kvaskoff M, Mu F, Terry KL et al (2015) Endometriosis: a highrisk population for major chronic diseases? Hum Reprod Update. https://doi.org/10.1093/humupd/dmv013

4. Parazzini F, Esposito G, Tozzi L, Noli S, Bianchi S (2017) Epidemiology of endometriosis and its comorbidities. Eur $\mathrm{J}$ Obstet Gynecol Reprod Biol 209:3-7. https://doi.org/10.1016/j. ejogrb.2016.04.021

5. Mu F, Rich-Edwards J, Rimm EB, Spiegelman D, Forman JP, Missmer SA (2017) Association Between Endometriosis and Hypercholesterolemia or Hypertension. Hypertension 70(1):59 65. https://doi.org/10.1161/hypertensionaha.117.09056

6. Melo AS, Rosa-e-Silva JC, Rosa-e-Silva AC, Poli-Neto OB, Ferriani RA, Vieira CS Unfavorable lipid profile in women with endometriosis. Fertil Steril 93(7):2433-2436. https://doi.org/10. 1016/j.fertnstert.2009.08.043

7. Verit FF, Erel O, Celik N (2008) Serum paraoxonase-1 activity in women with endometriosis and its relationship with the stage of the disease. Hum Reprod 23(1):100-104. https://doi.org/10.1093/ humrep/dem 340

8. Turgut A, Ozler A, Goruk NY, Tunc SY, Evliyaoglu O, Gul T (2013) Copper, ceruloplasmin and oxidative stress in patients with advanced-stage endometriosis. Eur Rev Med Pharmacol Sci 17(11):1472-1478

9. Pretta S, Remorgida V, Abbamonte LH et al (2007) Atherosclerosis in women with endometriosis. Eur J Obstet Gynecol Reprod Biol 132(2):226-231. https://doi.org/10.1016/j.ejogrb.2006.04.015

10. Mu F, Rich-Edwards J, Rimm EB, Spiegelman D, Missmer SA (2016) Endometriosis and Risk of Coronary Heart Disease. Circ Cardiovasc Qual Outcomes 9(3):257-264. https://doi.org/10.1161/ circoutcomes.115.002224

11. Harada T, Iwabe T, Terakawa N (2001) Role of cytokines in endometriosis. Fertil Steril 76(1):1-10

12. Lebovic DI, Mueller MD, Taylor RN (2001) Immunobiology of endometriosis. Fertil Steril 75(1):1-10

13. Agic A, Xu H, Altgassen $\mathrm{C}$ et al (2007) Relative expression of 1,25dihydroxyvitamin D3 receptor, vitamin D 1 alpha-hydroxylase, vitamin D 24-hydroxylase, and vitamin D 25-hydroxylase in endometriosis and gynecologic cancers. Reprod Sci 14(5):486-497. https://doi.org/10.1177/1933719107304565

14. Bedaiwy MA, Falcone T, Sharma RK et al (2002) Prediction of endometriosis with serum and peritoneal fluid markers: a prospective controlled trial. Hum Reprod 17(2):426-431 
15. Mu F, Harris HR, Rich-Edwards JW et al (2017) A Prospective Study of Inflammatory Markers and Risk of Endometriosis. Am J Epidemiol. https://doi.org/10.1093/aje/kwx272

16. Takemura Y, Osuga Y, Harada M et al (2005) Serum adiponectin concentrations are decreased in women with endometriosis. Hum Reprod 20(12):3510-3513

17. Bedaiwy MA, Falcone T, Goldberg JM, Sharma RK, Nelson DR, Agarwal A (2006) Peritoneal fluid leptin is associated with chronic pelvic pain but not infertility in endometriosis patients. Hum Reprod 21(3):788-791

18. Shah DK, Correia KF, Harris HR, Missmer SA (2013) Plasma adipokines and endometriosis risk: a prospective nested casecontrol investigation from the Nurses' Health Study II. Hum Reprod 28(2):315-321. https://doi.org/10.1093/humrep/des411

19. Centers for Disease Control and Prevention (2017) National Diabetes Statistics Report, 2017. Centers for Disease Control and Prevention, U.S. Dept of Health and Human Services, Atlanta, GA

20. Tobias DK, Chavarro JE, Williams MA et al (2013) History of infertility and risk of gestational diabetes mellitus: a prospective analysis of 40,773 pregnancies. Am J Epidemiol 178(8):12191225. https://doi.org/10.1093/aje/kwt110

21. Farland LV, Prescott J, Sasamoto N et al (2019) Endometriosis and Risk of Adverse Pregnancy Outcomes. Obstet Gynecol. https://doi. org/10.1097/aog.0000000000003410

22. Tobias DK, Gaskins AJ, Missmer SA et al (2015) History of infertility and risk of type 2 diabetes mellitus: a prospective cohort study. Diabetologia 58(4):707-715. https://doi.org/10.1007/s00125-0153493-z

23. Farland LV, Missmer SA, Bijon A et al (2017) Associations among body size across the life course, adult height and endometriosis. Hum Reprod 32(8):1732-1742. https://doi.org/10.1093/humrep/ $\operatorname{dex} 207$

24. Shah DK, Correia KF, Vitonis AF, Missmer SA (2013) Body size and endometriosis: results from 20 years of follow-up within the Nurses' Health Study II prospective cohort. Hum Reprod 28(7): 1783-1792. https://doi.org/10.1093/humrep/det120

25. Duleba AJ (1997) Diagnosis of endometriosis. Obstet Gynecol Clin N Am 24(2):331-346

26. Pardanani S, Barbieri RL (1998) The gold standard for the surgical diagnosis of endometriosis: visual findings or biopsy results? J Gynecol Tech 4:121-124

27. Johnson NP, Hummelshoj L, Adamson GD et al (2017) World Endometriosis Society consensus on the classification of endometriosis. Hum Reprod 32(2):315-324. https://doi.org/10.1093/ humrep/dew293

28. Missmer SA, Hankinson SE, Spiegelman D, Barbieri RL, Marshall LM, Hunter DJ (2004) Incidence of laparoscopically confirmed endometriosis by demographic, anthropometric, and lifestyle factors. Am J Epidemiol 160(8):784-796. https://doi.org/10.1093/ aje/kwh275

29. National Diabetes Data Group (1979) Classification and diagnosis of diabetes mellitus and other categories of glucose intolerance. Diabetes 28(12): 1039-1057. https://doi.org/10.2337/diab.28.12. 1039

30. The Expert Committee on the Diagnosis and Classification of Diabetes Mellitus (1997) Report of the expert committee on the diagnosis and classification of diabetes mellitus. Diabetes Care 20(7): 1183-1197. https://doi.org/10.2337/diacare.20.7.1183

31. Manson JE, Rimm EB, Stampfer MJ et al (1991) Physical activity and incidence of non-insulin-dependent diabetes mellitus in women. Lancet 338(8770):774-778

32. Correia KF, Dodge LE, Farland LV et al (2020) Confounding and effect measure modification in reproductive medicine research. Hum Reprod 35(5):1013-1018. https://doi.org/10.1093/humrep/ deaa 051
33. Farland LV, Correia KFB, Dodge LE et al (2020) The importance of mediation in reproductive health studies. Hum Reprod 35(6): 1262-1266. https://doi.org/10.1093/humrep/deaa064

34. McKinlay SM, Bifano NL, McKinlay JB (1985) Smoking and Age at Menopause in Women. Ann Intern Med 103(3): 350-356. https:// doi.org/10.7326/0003-4819-103-3-350\%m4026083

35. Missmer SA, Hankinson SE, Spiegelman D et al (2004) Reproductive history and endometriosis among premenopausal women. Obstet Gynecol 104(5 Pt 1):965-974. https://doi.org/10. 1097/01.AOG.0000142714.54857.f8

36. Nnoaham KE, Hummelshoj L, Webster P, et al. (2011) Impact of endometriosis on quality of life and work productivity: a multicenter study across ten countries. Fertil Steril 96(2): 366-373 e368. https://doi.org/10.1016/j.fertnstert.2011.05.090

37. Prescott J, Farland LV, Tobias DK et al (2016) A prospective cohort study of endometriosis and subsequent risk of infertility. Hum Reprod. https://doi.org/10.1093/humrep/dew085

38. Farland LV, Harris HR (2020) Long-Term Health Consequences of Endometriosis - Pathways and Mediation by Treatment. Curr Obstet Gynecol Rep 9(3):79-88. https://doi.org/10.1007/s13669020-00287-9

39. Guo C, Li Q, Tian G et al (2019) Association of age at menopause and type 2 diabetes: A systematic review and dose-response metaanalysis of cohort studies. Prim Care Diabetes 13(4):301-309. https://doi.org/10.1016/j.pcd.2019.02.001

40. Luo J, Manson JE, Urrutia RP, Hendryx M, LeBlanc ES, Margolis KL (2017) Risk of Diabetes After Hysterectomy With or Without Oophorectomy in Postmenopausal Women. Am J Epidemiol 185(9):777-785. https://doi.org/10.1093/aje/kwx023

41. Appiah D, Winters SJ, Hornung CA (2014) Bilateral Oophorectomy and the Risk of Incident Diabetes in Postmenopausal Women. Diabetes Care 37(3):725-733. https:// doi.org/10.2337/dc13-1986

42. Dørum A, Tonstad S, Liavaag AH, Michelsen TM, Hildrum B, Dahl AA (2008) Bilateral oophorectomy before 50 years of age is significantly associated with the metabolic syndrome and Framingham risk score: a controlled, population-based study (HUNT-2). Gynecol Oncol 109(3):377-383. https://doi.org/10. 1016/j.ygyno.2008.02.025

43. Rayanagoudar G, Hashi AA, Zamora J, Khan KS, Hitman GA, Thangaratinam S (2016) Quantification of the type 2 diabetes risk in women with gestational diabetes: a systematic review and metaanalysis of 95,750 women. Diabetologia 59(7):1403-1411. https:// doi.org/10.1007/s00125-016-3927-2

44. Tobias DK (2018) Prediction and Prevention of Type 2 Diabetes in Women with a History of GDM. Curr Diabetes Rep 18(10):78. https://doi.org/10.1007/s11892-018-1063-8

45. Bellamy L, Casas JP, Hingorani AD, Williams D (2009) Type 2 diabetes mellitus after gestational diabetes: a systematic review and meta-analysis. Lancet 373(9677):1773-1779. https://doi.org/10. 1016/s0140-6736(09)60731-5

46. Reaven GM (1988) Banting lecture 1988. Role of insulin resistance in human disease. Diabetes 37(12):1595-1607. https://doi.org/10. 2337/diab.37.12.1595

47. Kahn SE, Hull RL, Utzschneider KM (2006) Mechanisms linking obesity to insulin resistance and type 2 diabetes. Nature 444(7121): 840-846. https://doi.org/10.1038/nature05482

48. Rothman KJ, Sander G, Lash TL (2008) Modern epidemiology. 3 edn. Lippincott Williams \& Wilkins, Philidelphia

Publisher's note Springer Nature remains neutral with regard to jurisdictional claims in published maps and institutional affiliations. 